\title{
INTERPRETACIÓN MARKOVIANA DEL PROCESO DE ENVEJECIMIENTO Y MORTALIDAD
}

\author{
Roberto Ham Chande \\ El Colegio de México
}

\section{INTRODUCCIÓN}

LA INTENCIÓN de este trabajo es la de enmarcar el proceso del envejecimiento y mortalidad de un individuo dentro de la estructura matemática de una cadena finita de Markov. Para tal fin, se utilizan definiciones y resultados de la teoría probabilística correspondiente que tienen interpretación y aplicación inmediatas al proceso de vida y muerte de un individuo. ${ }^{x}$

\section{LA MORTALIDAD COMO UN PROCESO}

Se dice que un proceso es un fenómeno que cambia en función del tiempo, de tal modo que observar un proceso es fijarse en el estado que guarda el fenómeno al momento de la observación. Distinguimos entonces dos componentes esenciales en un proceso: el conjunto de los posibles estados del fenómeno, y el parámetro tiempo.

Como un ejemplo de un proceso se tiene lo siguiente: iniciar el fenómeno con un individuo que nace vivo, y vigilar las edades que alcanza hasta su muerte. Para este caso, las distintas edades y la condición de muerte son los posibles estados del fenómeno, mientras que el parámetro tiempo está dado por las fechas de las observaciones. A este fenómeno lo denominaremos proceso de envejecimiento y mortalidad, o simplemente proceso de mortalidad.

Siendo el ejemplo anterior el proceso que precisamente se pretende asimilar dentro del marco teórico que se propone, hacemos la siguiente idealización: observamos a un individuo que nace vivo, y cada año, en la fecha de su cumpleaños, y sólo en esa fecha, observamos lo que ha sucedido con él en el sentido de que continúe vivo con un año más de edad, o que haya muerto. Se supone además que lo máximo que podrá sobrevivir será hasta una edad entera que se denomina $\omega$.

Así entonces, se consideran los siguientes estados en el proceso de envejecimiento y muerte:

1 Tanto las definiciones y los teoremas relacionados directamente con la teoría de las cadenas finitas de Markov que aquí se citan pueden consultarse en el texto Finite Markov Chains de J. Kemeny y L. Snell, Princeton, D. Van Nostrand Co., 1960, donde se hacen claras demostraciones de los resultados, y las cuales no se exponen en este ensayo. 


$$
\begin{gathered}
E_{0}=\text { alcanzar vivo la edad } 0 \text { (nacer vivo), } \\
E_{1}:=\text { alcanzar vivo la edad } 1, \\
E_{2}=\text { alcanzar vivo la edad } 2, \\
\cdot \\
\cdot \\
E_{\omega}=\text { alcanzar vivo la edad } \omega \\
E_{\omega+1}=M=\text { morir. }
\end{gathered}
$$

Tales estados forman el conjunto finito $E=\left\{E_{0}, E_{1}, E_{2}, \ldots, E_{\omega}, E_{\omega+1}\right\}$ de los posibles estados del proceso.

Al mismo tiempo definimos otro conjunto $T$ que será el conjunto de los valores que podrá tomar el parámetro tiempo, y puesto que se ha convenido que las observaciones serán anuales, este conjunto $T$ será el de los años enteros, es decir $T=\{0,1,2, \ldots\}$.

Cada observación anual se dirá que es una etapa del proceso, y se identifican directamente con el conjunto $T$ de nuestro caso. De esta manera, si en cierta etapa se encuentra al proceso en el estado $E_{x}$, es decir, al individuo en observación vivo a edad entera $x$, en la siguiente etapa lo encontraremos ya sea en $E_{x+1}$ o en $M$, según que continúe vivo ahora con edad $x+1$, o que haya fallecido. Si en alguna etapa se encuentra al proceso en el estado $M$, invariablemente el proceso permanecerá ahí en todas las etapas posteriores.

Una de las herramientas para el estudio del fenómeno de la mortalidad la constituye lo que se denomina tabla de mortalidad, la cual refleja las condiciones del fenómeno en el momento, y estima para cada edad entera la probabilidad de sobrevivencia individual hasta la siguiente edad, y la de morir antes de alcanzar esa siguiente edad, en una población estacionaria hipotética. Tomando la tabla de mortalidad como base se tienen la siguiente notación:

$(x)=$ un individuo con vida $y$ exactamente $x$ años enteros de edad

$q_{x}=$ probabilidad de que $(x)$ muera antes de alcanzar la edad $x+1$

$p_{x}=\underset{\text { probabilidad de que }(x) \text { llegue con vida a }}{\text { la edad } x+1}$

${ }_{x} q_{x}=$ probabilidad de que $(x)$ muera antes de cumplir $x+k$ años de edad

${ }_{2} p_{x}=$ probabilidad de que $(x)$ sobreviva hasta la edad $x+k$

$e_{x}=$ esperanza de vida a la edad $x$, o número promedio de años que se espera que $(x)$ sobreviva. 


\section{LA MORTALIDAD COMO PROCESO ESTOCÁSTICO}

Se define como proceso estocástico un conjunto de distribuciones de probabilidad que dependen de cierto parámetro $x$. Se analiza en seguida cómo el proceso de mortalidad concuerda con esta definición:

Sea $f_{x}$ el resultado de la observación del proceso en la etapa $x$. Entonces $f_{x}$ es una variable aleatoria cuyos valores posibles son los estados del proceso, y la cual puede definirse como:

$$
f_{x}= \begin{cases}E_{x} & \text { si el individuo en observación vive a edad } x . \\ M & \text { si el individuo en observación ha muerto antes } \\ \text { de cumplir } x \text { años de edad. }\end{cases}
$$

Indicando con $\operatorname{Pr}\left(f_{x}=E_{i}\right)$ la probabilidad de que en la etapa $x$ el proceso se encuentre en el estado $E_{i}$ se va a tener que para cada $x$, el conjunto $\left\{\operatorname{Pr}\left(f_{x}=E_{i}\right)\right\}$ con $i=0,1,2, \ldots, \omega+1$ forma una distribución de probabilidad. En efecto, se tiene que:

Por convención el proceso se inicia en $E_{0}$, lo cual implica que en la etapa inicial, cuando $x=0$, se tendrá:

$$
\left\{\begin{array}{l}
\operatorname{Pr}\left(f_{0}=E_{0}\right)=1 \\
\operatorname{Pr}\left(f_{0}=E_{i}\right)=0
\end{array}\right.
$$

En etapas posteriores, cuando $x \neq 0$, se tienen los siguientes dos casos:

a) El individuo se encuentra con vida o edad $x-1$, es decir $f_{x-1}=$ $E_{x-1}$, teniéndose entonces que:

$$
\left\{\begin{array}{l}
\operatorname{Pr}\left(f_{x}=E_{x}\right)=p_{x-1} \\
\operatorname{Pr}\left(f_{x}=M\right)=q_{x-1} \\
\operatorname{Pr}\left(f_{x}=E_{i}\right)=0 \text { cuando } i \neq x \text { o cuando } i \neq \omega+1
\end{array}\right.
$$

b) E1 individuo en observación ha muerto antes de cumplir $x-1$ años de edad, esto es $f_{x-1}=M$, por lo cual:

$$
\left\{\begin{array}{l}
\operatorname{Pr}\left(f_{x}=M\right)=1 \\
\operatorname{Pr}\left(f_{x}=E_{i}\right)=0 \text { cuando } \mathrm{i} \neq \omega+1
\end{array}\right.
$$

En cualquiera de los casos anteriores para cada $x$ se tiene siempre que: $\sum_{i} \operatorname{Pr}\left(f_{x}=E_{i}\right)=1$, lo cual indica que en cada etapa $x$ cada estado $E_{i}$ tiene una probabilidad de ser alcanzado (ya sea positiva o cero), y estas probabilidades suman 1. Dicho de otra manera, tenemos una distribución de probabilidad sobre los estados en cada etapa $x$. Se concluye entonces que el proceso de mortalidad es un proceso estocástico puesto que se trata de un conjunto de distribuciones de probabilidad, de tipo binomial $\left\{\left(p_{x}, q_{x}\right)\right\}$ que dependen del parámetro $x$ que es la edad, lo cual está de acuerdo con la definición citada de lo que es un proceso estocástico. 


\section{LA MORTALIDAD COMO PROCESO MARKOVIANO}

Un proceso estocástico en el que $E$ y $T$ son discretos se dice que cumple la "condición de Markov" si para cualquier evento $S$ que ocurre antes de la etapa $x$ resulta que:

$$
\operatorname{Pr}\left(f_{x+1}=E_{i} / f_{x}=E_{j}, S\right)=\operatorname{Pr}\left(f_{x+1}=E_{i} / f_{x}=E\right)
$$

es decir, que el pasado no influye en los resultados futuros, sino que únicamente influirá la situación del proceso en el momento presente.

Por consideraciones ya hechas con anterioridad, en la construcción de la tabla de mortalidad se encuentra implícita la condición de Markov. Se ha supuesto que en el proceso que estudiamos las observaciones se hacen exacta y únicamente en el cumpleaños del individuo en cuestión, y tomando en cuenta únicamente si se encuentra vivo o no. Es entonces que dada una edad alcanzada, el pasado del individuo no es pertinente a sus probabilidades de vida y muerte en la etapa próxima, teniéndose así que la mortalidad es un proceso markoviano.

En un proceso que cumple la condición de Markov, la probabilidad de pasar del estado $E_{i}$ al estado $E_{j}$ en la etapa $x$ se denota como $p_{i j}(x)$, se denomina probabilidad de transición, y está definida por:

$$
p_{i j}(x)=\operatorname{Pr}\left(f_{x+1}=E_{j} / f_{x}=E_{i}\right)
$$

Cuando $p_{i j}(x)$ es independiente de la etapa $x$, esta probabilidad de transición se puede escribir sencillamente como $p_{i j}$. En el caso del proceso de la mortalidad, las probabilidades de transición se ven afectadas directamente por el valor de los índices $i j$ y no tanto por la etapa $x$, por lo cual las consideramos del tipo $p_{i j}$, independientes de $x$.

\section{La mortalidad como cadena finita de Markov}

Se dice que una cadena finita de Markov es una terna $(E, \pi, R)$ donde:

a) $E=\left\{E_{1}, E_{2}, \ldots, E_{n}\right\}$ es el conjunto finito de los $n$ posibles estados del proceso.

b) $\pi=\left\{\pi_{1}, \pi_{2}, \ldots, \pi_{n}\right\}$ es un vector probabilístico, donde $\pi_{i}$ es la probabilidad de que en la etapa inicial el proceso se encuentre en $E_{i}$.

c) $R$ es una matriz cuadrada de orden $n$, que se denomina matriz de transiciones, y que está compuesta precisamente por las probabilidades de transición las cuales son independientes de la etapa, es decir,

$$
R=\left[\begin{array}{cccc}
p_{11} & p_{12} & \ldots & p_{1 n} \\
p_{22} & p_{222} & \ldots & p_{2 n} \\
\ldots & \ldots & \ldots & \ldots \\
p_{n 1} & p_{n 2} & \cdots & p_{n n}
\end{array}\right]
$$

y $p_{i j}=\operatorname{Pr}\left(f_{x+1}=E_{j} / f_{x}=E_{i}\right)$ independientemente de $x$. 
Se observa en seguida que el proceso estocástico de la mortalidad es una cadena finita de Markov desde el momento en que se puede definir de la siguiente manera:

El proceso de la mortalidad es una pareja $(E, R)$ donde:

a) $E=\left\{E_{0}, E_{1}, E_{2}, \ldots, E_{\omega}, E_{\omega+1}\right\}$, considerando a $E_{i}$ de acuerdo con la definición que dimos de los estados del proceso, es decir, las edades de 0 a $\omega$ y el estado de muerte. Tenemos entonces $n=$ $\omega+2$ como el número de estados posibles.

b) $R$ es una matriz cuadrada de orden $n=\omega+2$, donde cada uno de sus elementos $p_{i j}$ se define de la siguiente manera:

$$
p_{i j}= \begin{cases}1 & \text { para }\left\{\begin{array}{l}
i=1 \\
j=1
\end{array}\right. \\
q_{i-2} & \text { para }\left\{\begin{array}{l}
i=2,3, \ldots, \omega+2 \\
j=1
\end{array}\right. \\
p_{i-2} & \text { para }\left\{\begin{array}{l}
i=2,3, \ldots, \omega+2 \\
j=i+1
\end{array}\right. \\
0 & \text { para cualesquiera otros índices } i j .\end{cases}
$$

donde $p_{x}$ y $q_{x}$ significan las probabilidades de sobrevivencia y muerte en un año más teniendo la edad actual $x$, tal y como se definieron al comienzo. Además se tomaron en cuenta las siguientes consideraciones adicionales:

a) El renglón y la columna 1 se refieren al estado $M$.

b) El renglón y la columna $i$, con $i=2,3, \ldots, \omega+2$ se refieren al estado $E_{i-2}$, o sea al de alcanzar vivo la edad $i-2$

Para aclarar la forma de $R$ la escribimos directamente en forma matricial, agregando en los márgenes los índices y estados a los que corresponden columnas y renglones:

$$
\begin{aligned}
& i=\begin{array}{lllllc}
1 & 2 & 3 & 4 & \ldots & \omega+2 \\
M & E_{0} & E_{1} & E_{2} & \ldots & E \omega
\end{array} \quad j= \\
& R=\left[\begin{array}{cccccc}
1 & 0 & 0 & 0 & \ldots & 0 \\
q_{0} & 0 & p_{0} & 0 & \cdots & 0 \\
q_{1} & 0 & 0 & p_{1} & \cdots & 0 \\
. & . & . & . & . & . \\
. & . & . & . & . & . \\
. & . & . & . & . & . \\
q_{\omega-1} & 0 & 0 & 0 & \cdots & p_{\omega-1} \\
q_{\omega} & 0 & 0 & 0 & \cdots & 0
\end{array}\right] \quad \begin{array}{ccc}
M & 1 \\
E & . & . \\
E_{\omega-1} & \omega+1 \\
E_{\omega} & \omega+2
\end{array}
\end{aligned}
$$

Dicho de otro modo, $R$ no es más que la tabla de mortalidad escrita en una forma matricial peculiar; donde sólo se utilizan las probabilidades $p_{x}$ y $q_{x}$, agregándose el concepto del estado $M$ con la obvia conside- 
ración de que al llegar el proceso a tal estado permanecerá indefinidamente ahí con probabilidad 1.

En la definición de lo que es una cadena finita de Markov interviene un vector probabilístico $\pi$. Sin embargo, al definirse a la mortalidad como cadena finita de Markov no se tomó en cuenta tal vector. Esto último fue debido a la consideración de que el proceso se inicia al comenzar la observación de un individuo que nace vivo. Es decir, que en la etapa 0 el proceso se encuentra en $E_{0}$, siendo entonces que para este caso particular $\pi$ será siempre de la forma $(0,1,0,0, \ldots, 0)$.

\section{Probabilidades de sobrevivencia y muerte en $k$ años}

Dentro de la matemática de las cadenas finitas de Markov tenemos el siguiente teorema:

Si $S_{i}$ y $S_{j}$ representan respectivamente los estados que se refieren al i-ésimo renglón y a la j-ésima columna de $R$, entonces

$$
\operatorname{Pr}\left(f_{x+k}=S_{j} / f_{x}=S_{i}\right)=\left(R^{k}\right)_{i i}
$$

Esto es, la probabilidad de ir del estado $S_{i}$ al estado $S_{j}$ en exactamente $k$ etapas más, es la componente $i j$ de la matriz $R^{k}$.

El anterior resultado nos indica que al elevar $R$ a la $k$, se obtienen las probabilidades de que $(x)$ sobreviva hasta la edad $x+k$, y de que muera antes de cumplir $x+k$ años de edad. Manteniendo a $k$ entre 0 y $\omega$ corroboramos la anterior afirmación al analizar la forma de $R^{k}$ :

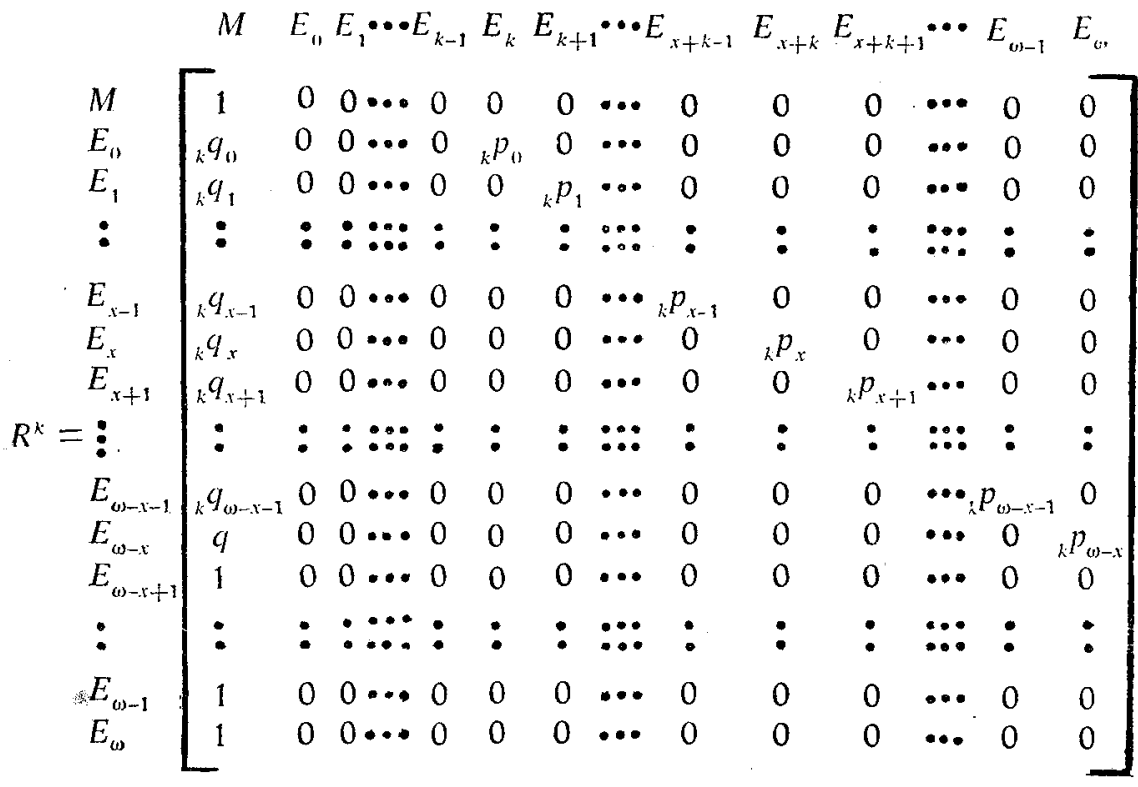




\section{CONVERgENCIA DEL PROCESO}

Observando el resultado anterior, de haber elevado $R$ a la $k$, notamos que cuando $x \geq \omega-x+1$, la probabilidad de llegar a $M$ desde cualquiera de los estados $E_{x}, E_{x+1} \ldots, E_{\omega} \circ M$ es exactamente 1. Esto último no es más que la consecuencia lógica del supuesto inicial de que nadie sobrevive más allá de los $\omega$ años. Cuando $k=\omega+1$ tendremos. entonces:

$$
R^{\omega+1}=\left(\begin{array}{ccccccc}
1 & 0 & 0 & 0 & . & . & 0 \\
1 & 0 & 0 & 0 & . & . & 0 \\
1 & 0 & 0 & 0 & . & . & 0 \\
1 & 0 & 0 & 0 & . & . & 0 \\
. & . & . & . & . & . & . \\
. & . & . & . & . & . & . \\
. & . & . & . & . & . & . \\
1 & 0 & 0 & 0 & . & . . & 0
\end{array}\right)
$$

y además es algebraicamente simple notar que $R^{\omega+2}=R^{\omega+1} R=R^{\omega+1}$ lo cual indica que $R^{\omega+1}=R^{\omega+2}=R^{\omega+3}=\ldots$ $\mathrm{y}$ esto último se puede escribir como

$$
R^{k} \rightarrow\left(\begin{array}{cccccc}
1 & 0 & 0 & . & \ldots & 0 \\
1 & 0 & 0 & . & . & 0 \\
1 & 0 & 0 & . & . . & 0 \\
. & . & . & . & . & . \\
. & . & . & . & . & . \\
. & . & . & . & . & . \\
1 & 0 & 0 & . & . & 0
\end{array}\right) \text { cuando } k \rightarrow \infty
$$

lo cual no es más que el resultado obvio de que la probabilidad de morir teniendo cualquier edad es 1 si se da un plazo $k$ de años lo suficientemente grande.

\section{LA MORTALIDAD COMO CADENA ABSORBENTE}

Se hace ahora mención de tres definiciones, con el mismo concepto en la notación $S_{i}$ que en el punto anterior:

a) El estado $S_{i}$ se llama estado de transición si existe al menos una $j \neq i$ para la cual $p_{i j}>0$. Es decir, que si el proceso llega a alcanzar el estado $S_{i}$, se tiene una probabilidad positiva de salir de él.

b) El estado $S_{i}$ se denomina estado absorbente si $p_{i j}=1$. Es decir, que cuando el proceso llega a tal estado jamás sale de ahí.

c) Una cadena finita de Markov se llama absorbente si tiene al menos un estado absorbente. 

estados:

En una cadena absorbente de Markov distinguimos dos conjuntos de

$$
\begin{aligned}
& A=\{\text { estados absorbentes } \\
& B=\text { estados de transición }
\end{aligned}
$$

Tenemos entonces que el proceso de mortalidad es una cadena absorbente donde $A=\{M\}$, hay un solo estado absorbente que es $M, \mathrm{y}$ $B=\left\{E_{0}, E_{1}, \ldots, E_{\omega}\right\}$, en el que los estados de transición son todas las posibles edades.

En la matriz $R$ hagamos la siguiente partición respecto a los conjuntos $A$ y $B$ anteriores:

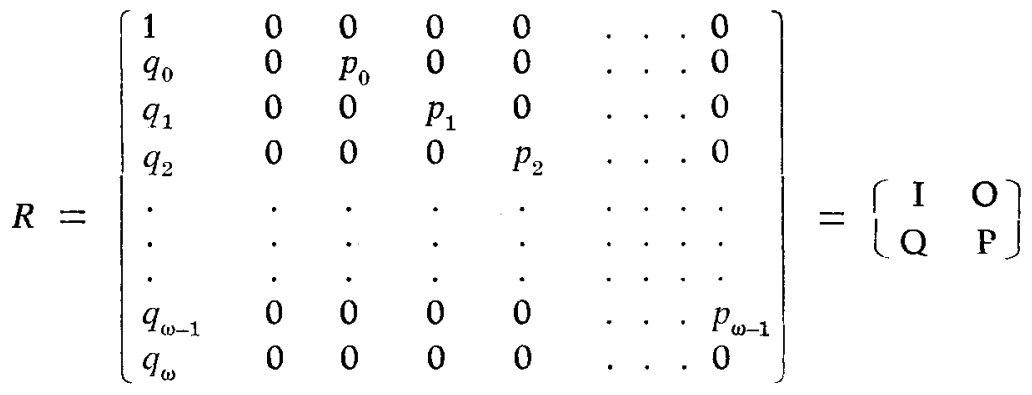

definiéndose las siguientes matrices:

$$
\begin{aligned}
& I=[1] \quad O=\left[\begin{array}{ll}
0,0,0, \ldots, 0
\end{array}\right]
\end{aligned}
$$

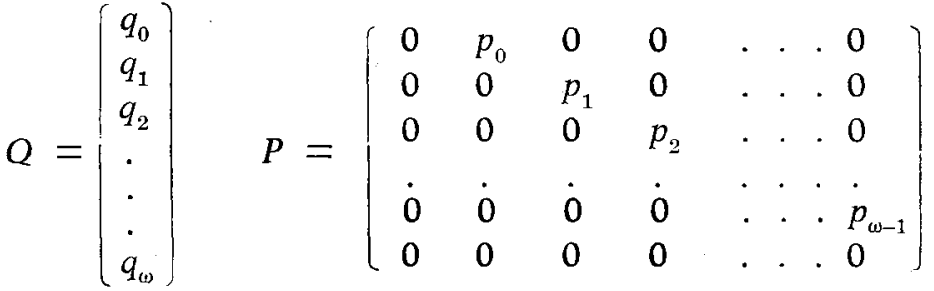

$I$ - implica que una vez alcanzado el estado $M$ el proceso continuará ahí con probabilidad 1.

$O$ - indica que una vez en el estado $M$, la probabilidad de ir a cualquier otro estado $E_{x}$ es 0 .

$Q$ - indica las probabilidades de pasar de un estado de transición al estado absorbente en una etapa más, es decir, las probabilidades de que estando vivo sobrevenga la muerte dentro del siguiente año.

$P$ - señala las probabilidades de pasar de un estado de transición a otro de transición en la etapa siguiente, o dentro de la presente interpretación, la probabilidad de que estando vivo se continúe con vida al año siguiente.

IX. Probabilidades de supervivencia de edad $x_{i}$ a edad $x_{j}$

Se cita el siguiente resultado general de las cadenas absorbentes de Markov, para posteriormente enfocarlo hacia una aplicación dentro de nuestro proceso en especial: 
Si $E_{x}$ y $E_{y}$ son estados de transición y $h_{x y}$ es el número de veces que la cadena pasa por $E_{y}$ suponiendo que la observación inicial fue $E_{x}$, no necesariamente al principio del proceso, tendremos:

$$
m\left(h_{x y}\right)=[I-P]_{x y}^{-1}
$$

es decir, la media de la variable aleatoria $h_{x y}$ es la componente $x y$ de la matriz inversa de $I-P$.

Se busca ahora la interpretación de este resultado para el proceso de mortalidad. Dado que $(x)$ a lo más puede pasar una sola vez por una edad $y \geq x$, la media del número de veces que el proceso pasa por $E_{y}$ habiendo partido de $E_{x}$ se convierte propiamente en una frecuencia relativa, o lo que es lo mismo, en una probabilidad, siendo entonces que la matriz $H=[I-P]^{-1}$ está compuesta por las probabilidades de que $(x)$ alcance vivo la edad $y$.

Si se denomina por $H_{x y}$ la componente $x y$ de la matriz $H$, tendremos:

a) $H_{x y}=1$ puesto que la probabilidad de que $(x)$ alcance vivo precisamente la edad $x$ es obviamente 1 .

b) $H_{x y}=0$ cuando $y<x$ pues la probabilidad de retroceder en la edad es 0 .

c) $H_{x y}={ }_{y-x} p_{x}$ cuando $y>x$ dado que la probabilidad de que $(x)$ alcance vivo la edad $y$ es la probabilidad de que sobreviva años $y-x$ más.

Las condiciones anteriores se comprueban al calcularse la matriz: $H$ :

$\begin{array}{lllllllll}E_{0} & E_{1} & E_{2} & E_{3} & \ldots & E_{x} & \ldots & E_{\omega-1} & E_{\omega}\end{array}$

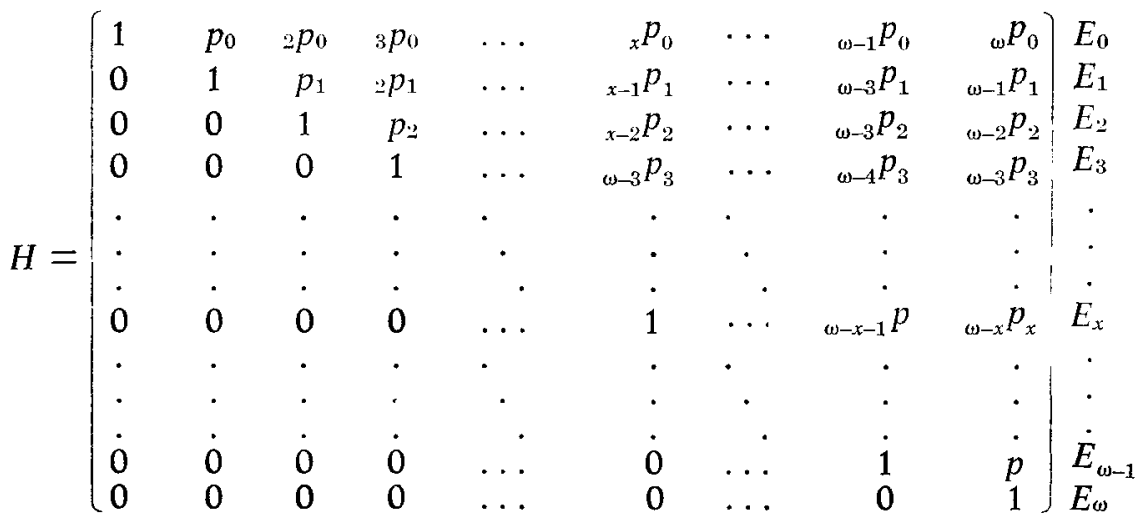

\section{Vector DE Esperanzas DE VIDA}

Estudiamos ahora el siguiente teorema:

Si $E_{x}$ es un estado de trancisión donde se inicia una observación, no necesariamente el inicio del proceso, $\nu_{x}$ es una variable aleatoria que señala el número de etapas que la cadena pasa antes de ser absorbida, descontándose la etapa de la observación inicial, entonces la media de $\nu_{x}$ 
está dada por la componente $x$-ésima del vector $H d$ menos 1 , donde $d=(1,1,1, \ldots, 1)^{\prime}$, es decir:

$$
m\left(\nu_{x}\right)=[H d]_{x}-1
$$

En el proceso de mortalidad cada etapa se refiere al transcurso de un año, siendo entonces que el número de etapas $\nu_{x}$ que el proceso recorre antes de ser absorbido, esto es, antes de llegar a $M$, iniciándose en $E_{x}$, es decir, a edad $x$, representan el número de años de vida que restan a $(x)$. En este caso $m\left(\nu_{x}\right)$ es la esperanza de vida $e_{x}$ a edad $x$.

Es fácil verificar la afirmación anterior al calcular el elemento $x$ del vector columna $H d$ :

$$
[H d]_{x}=1+\sum_{i=1}^{\omega-x}{ }_{i} p_{x}
$$

recordando que $\sum_{i=1}^{\omega-x}{ }_{i} p_{x}=e_{x}$, se puede escribir:

$$
[H d]_{x}-1=e_{x}
$$

Como punto final, se define vector $e$ de esperanzas de vida.

$$
e=\left(e_{0}, e_{1}, e_{2}, \ldots, e_{\omega}\right)
$$

y cuya expresión matricial, desde el punto de vista del proceso estocástico que hemos manejado, será:

$$
e=H d-d=(H d-I) d
$$

\section{Conclusiones}

Una cadena finita de Markov es la historia de un sistema que en el transcurso del tiempo sufre transiciones aleatorias de tipo discreto y finito, y que sin olvidarse totalmente su pasado, sólo le importa para su futuro el suceso más reciente. Tal historia está descrita totalmente por la terna $(E, \pi, R)$. En la forma de cadena de Markov, la narración sobre el proceso de mortalidad está dada por completo en la pareja $(E, R)$ donde $E$ señala el conjunto de las diversas edades que pueden ser alcanzadas además de la condición de muerte, y $R$ es la tabla de mortalidad escrita en una peculiar forma matricial. Esta matriz $R$ puede denominarse con toda propiedad como la matriz fundamental del sistema pues contiene implícitamente gran información sobre el proceso en estudio:

a) $R^{k}$ indica las probabilidades de que $(x)$ alcance con vida la edad $x+k$ y de que muera dentro de los próximos $k$ años.

b) Cuando $k \rightarrow \infty, R^{k}$ expresa que $(x)$ indefectiblemente morirá con probabilidad 1 , es decir, el proceso converge.

c) Haciendo la partición:

$$
R=\left[\begin{array}{ll}
I & O \\
Q & P
\end{array}\right]
$$


$Q$ señala las probabilidades de muerte dentro del próximo año, y $P$ las de sobrevivencia en un año más.

d) $\mathrm{H}=(I-P)^{-1}$ indica las probabilidades de que $(x)$ alcance con vida la edad $y$.

e) Definiendo $d=(1,1, \ldots, 1)^{\prime}$ se encuentra con que $e=(H-I) d$ es el vector compuesto por las esperanzas de vida.

Fuera del tratamiento markoviano que se le ha dado al proceso, los puntos meramente demográficos $\mathrm{y}$ actuariales tocados son apenas una mínima parte del principio. Sin embargo, se ha podido construir una estructura que esquematiza con rigor definiciones y conceptos dentro del proceso de mortalidad. Cabe ahora explorar las posibilidades de aplicaciones prácticas del modelo propuesto, ${ }^{2}$ y la de su extensión a un modelo markoviano de tipo continuo.

2 El autor, junto con Tomás Tello Sandoval, ha encontrado una aplicación al cálculo de primas netas de seguros de vida individuales: Tomás Tello Sandoval, Consideraciones markovianas sobre el seguro de vida individual, Tesis profesional, UNAM, 1969. 